\title{
Toxicities Associated with Docetaxel-Based Regimens in the Adjuvant Treatment of Early-Stage Breast Cancer: A Multicenter Prospective Real-Life Experience
}

\author{
Elie El Rassy ${ }^{a} \quad$ Marwan Ghosn $^{a} \quad$ Fadi Farhat $^{a, b} \quad$ Ziad Bakouny $^{a} \quad$ Tarek Assi $^{a}$ \\ Georges Chahine $^{\mathrm{a}, \mathrm{c}}$ Fadi Nasr ${ }^{\mathrm{a}, \mathrm{d}}$ Fady Ghassan Haddad ${ }^{\mathrm{a}}$ Fadi El Karak $^{\mathrm{a}}$ Joseph Kattan $^{\mathrm{a}, \mathrm{e}}$ \\ a Department of Hematology-Oncology, Hotel-Dieu de France University Hospital, Faculty of Medicine, \\ Saint Joseph University, Beirut, Lebanon; \\ ${ }^{b}$ Department of Medical Oncology, Hammoud Hospital, Saida, Lebanon; \\ ${ }^{c}$ Department of Medical Oncology, Notre Dame de Secours Hospital, Byblos, Lebanon; \\ dDepartment of Medical Oncology, Mount Lebanon Hospital, Hadath, Lebanon; \\ e Department of Medical Oncology, Saint Joseph Hospital, Baouchrieh, Lebanon
}

\section{Keywords}

Docetaxel - Breast cancer - Adverse events - Safety · Toxicity

\section{Summary}

Background: The present study reports on the efficacy and safety of adjuvant docetaxel in real-life patients with early-stage breast cancer. Methods: This is a prospective, multicenter, post-marketing study that evaluates the efficacy and safety of docetaxel-based regimens in patients with early breast cancer treated between 2007 and 2012. Results: A total of 698 female breast cancer patients receiving adjuvant docetaxel-based regimens were included in this study. Docetaxel monotherapy was administered in $4.2 \%$, whilst most patients received polychemotherapy. Non-hematological adverse events included skin reactions in $32.7 \%$ of the subjects. Multiple adverse events were reported and most commonly included asthenia (66.5\%), alopecia (43.4\%), and diarrhea $(24.2 \%)$. It is noteworthy that no fatal toxicities occurred. Several hematological adverse events were reported during treatment, with anemia being the most common. Conclusion: The results of this real-life experience, characterized by a relatively large sample size and long follow-up, confirm that docetaxel is effective and well tolerated in early-stage breast cancer patients.

(C) 2018 S. Karger GmbH, Freiburg

\section{Introduction}

Docetaxel is an active member of the taxane family which has shown efficacy in breast cancer across the metastatic, adjuvant, and neoadjuvant settings. It is a semi-synthetic agent which was first identified in 1986 as an alternative to paclitaxel [1]. Today, docetaxel constitutes one of the major treatment options targeting breast cancer. However, its clinical benefit is accompanied by intolerable adverse effects among which are neutropenia, fluid retention syndrome, hypersensitivity reactions, and gastrointestinal and mucocutaneous toxicities [2]. The present study focuses on adjuvant docetaxel in real-life breast cancer patients. Such data is of particular interest to ensure safety and good clinical practice in the use of the concerned drug. Our primary objective was to evaluate the efficacy and safety of docetaxel in the adjuvant setting for patients with breast cancer, including an evaluation of the adverse effects profile.

\section{Methods}

This is a prospective, multicenter, post-marketing study that evaluates the efficacy and safety of docetaxel-based adjuvant regimens in patients with early breast cancer.

\section{Study Population}

This study collected data on early-stage cancer patients treated between July 2007 and December 2012 with a docetaxel-based regimen in the adjuvant setting in 5 centers in Lebanon. The inclusion visit documented relevant patient history, details on diagnostic procedures, and planned therapy. The administered treatment was documented, as well as factors that influenced any change

\section{KARGER}

(c) 2018 S. Karger GmbH, Freiburg
Tarek Assi, MD

Department of Hematology-Oncology, Hotel-Dieu de France University Hospital

Faculty of Medicine, Saint-Joseph University

Beirut, Lebanon

tarekassi@gmail.com 
from the initial therapy plan in addition to the patient's status at the end of treatment and at each follow-up visit.

Patients eligible for this study had histologically confirmed breast adenocarcinoma with an ECOG performance status of $\leq 2$. Patients had to be suitable for a docetaxel-based regimen in the adjuvant setting (stage I, II, III) without prior chemotherapy. Adequate bone marrow, hepatic, and renal function was confirmed by hemoglobin $\geq 10 \mathrm{~g} / \mathrm{dl}$, absolute neutrophil count (ANC) $\geq 2,000 / \mathrm{mm}^{3}$, platelets $\geq 100,000 / \mathrm{mm}^{3}$, total bilirubin $\leq 1$ upper limit of normal (ULN), SGOT (AST) and SGPT (ALT) $\leq 2.5 \mathrm{UNL}$, and creatinine clearance $\geq 30 \mathrm{ml} / \mathrm{min}$. All patients included in the study had to sign an informed consent approved by the ethics committee of the Faculty of Medicine of Saint Joseph University.

We excluded pregnant and lactating women and patients with stage IV breast cancer, renal dysfunction (creatinine clearance $<30 \mathrm{ml} / \mathrm{min}$ ), total bilirubin $>1 \mathrm{UNL}$ or liver enzymes $>2.5 \mathrm{UNL}, \mathrm{ANC}<2,000 / \mathrm{mm}^{3}$ and platelet count $<100,000 / \mathrm{mm}^{3}$, and other prior malignancies within the past 5 years (except for cured basal cell carcinoma of the skin and excised carcinoma in situ of the cervix). Evidence of neurological disturbance or cerebral metastases, active peptic disease, and a personal history of inflammatory bowel disease were also among the exclusion criteria. Patients with current peripheral neuropathy $\geq$ grade 2 according to National Cancer Institute (NCI) criteria were also excluded. Patients who received neoadjuvant chemotherapy were not included in this analysis.

\section{Treatment}

All eligible patients were treated with docetaxel-based regimens either as monotherapy or in combination with other cytotoxic drugs. Treatments were administered according to the physicians' current practice, clinical guidelines, and ethical considerations. Radiation therapy was performed after administering adjuvant chemotherapy in patients who underwent partial mastectomy and axillary dissection with lymph node involvement. All tumors underwent immunohistochemical analysis for the evaluation of the hormone receptor (HR) and HER2 status. Patients with estrogen receptors (ER) or progesterone receptors (PR) scoring above $1 \%$ according to the Allred score received 5 years of aromatase inhibitors if they were postmenopausal or a selective ER modulator if they were premenopausal. Patients with positive HER2 status received trastuzumab for 1 year postoperatively, and those with equivocal HER2 underwent fluorescence in situ hybridization (FISH) analysis and were treated accordingly.

\section{Efficacy Assessment}

Follow-up was performed every 3 months during the first 2 years, then every 6 months until the 5th year, and then every year. Patients were physically examined by their primary physician at each visit. Laboratory tests were performed at each visit. Mammography and breast ultrasound were conducted every year. Abdominal imaging and bone scan were recommended as clinically indicated.

The primary endpoint of the study was to assess the efficacy and safety of docetaxel-based regimens in the adjuvant setting for patients with early breast cancer. Secondary endpoints included evaluation of commonly used regimens, safety profile, disease-free survival (DFS), and overall survival (OS). Survival was assessed from the time of enrollment until death from any cause.

\section{Safety Assessment}

All treated patients were included in the safety analysis for adverse events, laboratory abnormalities, vital signs, and time of onset of adverse events. Blood count as well as renal and liver function were recorded at the time of enrollment and at day 1 of each cycle. Blood count, serum creatinine, and side effects were recorded at each cycle and graded according to NCI-CTCAE version 3.0. All stage 4 or unexpected side effects were reported to the study coordinator.

\section{Statistical Analysis}

Data from all the participating centers were analyzed as one dataset. Qualitative variables were summarized using descriptive statistics and were reported as frequencies and percentages. Quantitative variables were presented as numbers, means, and standard deviations, minima and maxima. DFS and OS were compared across the levels of independent categorical variables using a KaplanMeier analysis followed by pairwise log-rank tests. In order to establish the determinants of the occurrence and increased severity of adverse events, multino-
Table 1. Patient $(\mathrm{n}=698)$ and cancer baseline characteristics

\begin{tabular}{ll}
\hline Baseline characteristics & \\
\hline Age, years & \\
$\quad$ Median & 51.8 \\
$\quad$ Range & $25.3-82.6$ \\
Performance status, \% & \\
0 & 93.3 \\
1 & 6.0 \\
2 & 0.7 \\
Stage, \% & \\
I & 13.6 \\
IIA & 34.1 \\
IIB & 28.8 \\
IIIA & 16.6 \\
IIIB & 6.7 \\
HR status, \% & \\
ER-/PR- & 49.0 \\
ER+/PR+ & 39.3 \\
ER+/PR- & 9.5 \\
ER-/PR+ & 2.3 \\
HR and HER2 status, \% & \\
HR+/HER2+ & 12.8 \\
HR+/HER2- & 39.0 \\
HR-/HER2+ & 17.3 \\
HR-/HER2- & 30.9 \\
\hline
\end{tabular}

$\mathrm{HR}=$ Hormone receptor; ER = estrogen receptor; $\mathrm{PR}=$ progesterone receptor.

mial stepwise regression models were established with adverse event severity as the dependent variable and disease as well as patient characteristics as independent variables. All statistical analyses were performed using IBM SPSS Statistics for Windows, version 22.0 (IBM Corp., Armonk, NY, USA; released 2013) and XIStat version 2017.1 (Addinsoft, Paris, France).

\section{Results}

\section{Patients}

A total of 698 female breast cancer patients receiving adjuvant docetaxel-based regimens were included in this study. The median age of the study population was 51.8 years (range $25.3-82.6$ years). The median patient follow-up was 32.88 months (range $0.2-187.0$ months). At diagnosis, $76.5 \%$ of the patients had either stage I, IIA, or IIB, and $95.1 \%$ had unilateral breast cancer with 50.8 and $44 \%$ in the left and right breast, respectively. The majority of patients (99.3\%) had a performance status of $\leq 1$. Overall, HRs were present in 48.6 and $41.5 \%$ for ER and PR, respectively. The subjects' breast cancers had the following hormonal phenotype: $49.0 \%$ ER-negative/PR-negative, 39.3\% ER-positive/PR-positive, 9.5\% ER-positive/ PR-negative, and 2.3\% ER-negative/PR-positive. HER2 was present in $28.0 \%$ of the patients being tested. Overall, $18.5 \%$ of our patients had equivocal HER2 status requiring further FISH analysis, among which $39.5 \%$ were FISH-positive, $28.7 \%$ were FISH-negative, and $21.8 \%$ did not undergo the test. Subsequently, the distribution of breast cancer cases was as follows: $12.8 \%$ HR-positive/HER2-positive, 39.0\% HR-positive/HER2-negative, 17.3\% HR-negative/ HER2-positive, and 30.9\% HR-negative/HER2-negative (table 1). 


\section{Efficacy}

Among our patients, 39 (5.6\%) did not complete the treatment as initially planned because of disease progression (7.7\%), adverse event occurrence $(10.3 \%)$, death (2.6\%), financial reasons $(2.6 \%)$, loss to follow-up (64.1\%), and other reasons (12.8\%). The median number of docetaxel injections was 4 (range 1-4). Docetaxel monotherapy was administered in $4.2 \%$ of cases; however, most patients received polychemotherapy with the following combinations being the most commonly used: docetaxel + adriamycin + cyclophosphamide (50.7\%), docetaxel + cyclophosphamide (27.9\%), docetaxel + adriamycin + cyclophosphamide +5 -fluorouracyl (10.2\%), and docetaxel + adriamycin (3.9\%) (table 2).

Recurrences occurred mostly at distant sites (84.3\%): liver (40.0\%), bone (38.5\%), and lung (33.3\%) (table 2). Rates of DFS at 3 and 5 years were $91.9 \%$ (95\% confidence interval (CI) $89.3-$ 94.4\%) and $82.3 \%$ (95\% CI 78.0-86.6\%), respectively. Mean DFS was 81.2 months (95\% CI 78.2-84.3 months) and was found to be similar $(\mathrm{p}=0.304)$ between patients who had completed the planned adjuvant treatment and those who had not (figs. 1, 2). Mean DFS was found to be independent of nodal status; in particu-

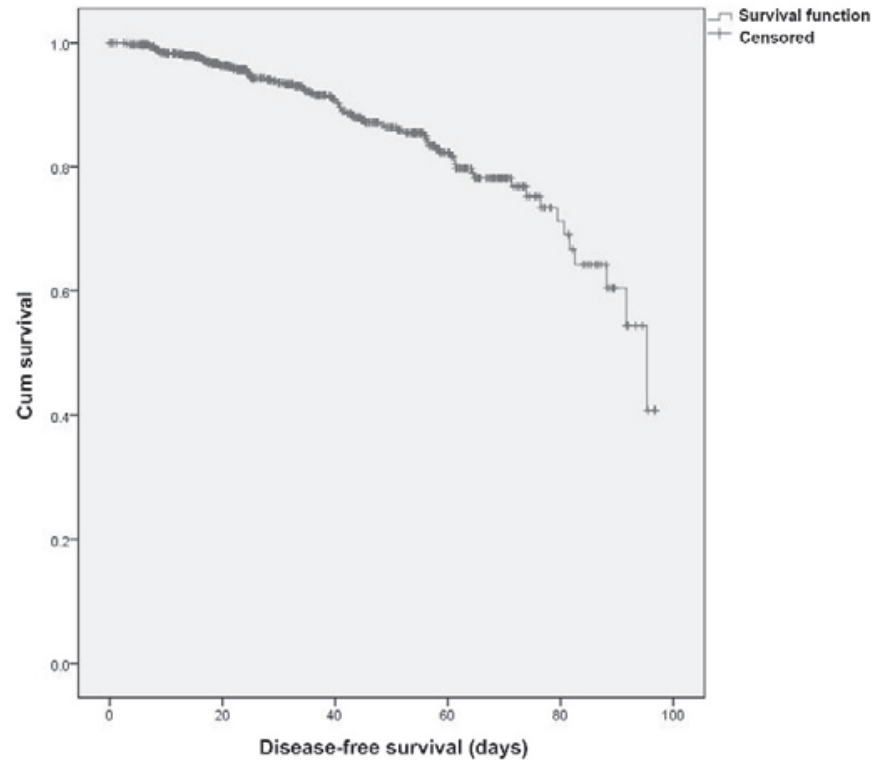

Fig. 1. Disease-free survival assessed by Kaplan-Meier in Lebanese breast cancer patients. lar, those with N1 disease had similar DFS compared to those with either $\mathrm{N} 2$ or $\mathrm{N} 3$ disease $(\mathrm{p}=0.994)$.

Rates of OS at 3 and 5 years were $98.8 \%$ (95\% CI 97.8-99.8\%) and $97.7 \%$ (95\% CI 96.3-99.0\%), respectively. Mean OS was found to be 116.7 months (95\% CI 78.2-84.3 months) (fig. 3) and significantly longer $(\mathrm{p}<0.001)$ in patients who had completed the planned adjuvant treatment (118.3 months; 95\% CI 117.3-119.2 months) compared to those who had not (73.3 months; $95 \%$ CI

Table 2. Docetaxel-based regimen in adjuvant breast cancer patients $(\mathrm{n}=698)$

\begin{tabular}{|c|c|}
\hline \multicolumn{2}{|l|}{ Treatment characteristics } \\
\hline Completed treatment, $\%$ & 95.1 \\
\hline \multicolumn{2}{|l|}{ Discontinuation of treatment, $\%$} \\
\hline Total & 4.9 \\
\hline Discontinuation for adverse events & 10.3 \\
\hline Discontinuation for disease progression & 7.7 \\
\hline Discontinuation for financial reasons & 2.6 \\
\hline Discontinuation for other reasons & 12.8 \\
\hline Death & 2.6 \\
\hline Loss to follow-up & 64.1 \\
\hline \multicolumn{2}{|l|}{ Injections, $n$} \\
\hline Median & 4 \\
\hline Range & $1-14$ \\
\hline \multicolumn{2}{|l|}{ Regimens, \% } \\
\hline Docetaxel + doxorubicin + cyclophosphamide & 50.7 \\
\hline Docetaxel + cyclophosphamide & 27.9 \\
\hline $\begin{array}{l}\text { Docetaxel }+ \text { doxorubicin }+ \text { cyclophosphamide }+ \\
\text { 5-fluorouracil }\end{array}$ & 10.2 \\
\hline Docetaxel monotherapy & 4.2 \\
\hline Docetaxel + doxorubicin & 3.9 \\
\hline Other & 3.1 \\
\hline \multicolumn{2}{|l|}{ Site of recurrence $(n=89), n(\%)$} \\
\hline Local & $16(18.0)$ \\
\hline Contralateral & $5(5.6)$ \\
\hline Distant & $75(84.3)$ \\
\hline Liver & $30(40.0)$ \\
\hline Lung & $25(33.3)$ \\
\hline Bone & $29(38.5)$ \\
\hline Lymph node & $15(20.0)$ \\
\hline Brain & $12(16.0)$ \\
\hline Other & $8(10.5)$ \\
\hline
\end{tabular}

Fig. 2. Mean diseasefree survival in patient subpopulations.

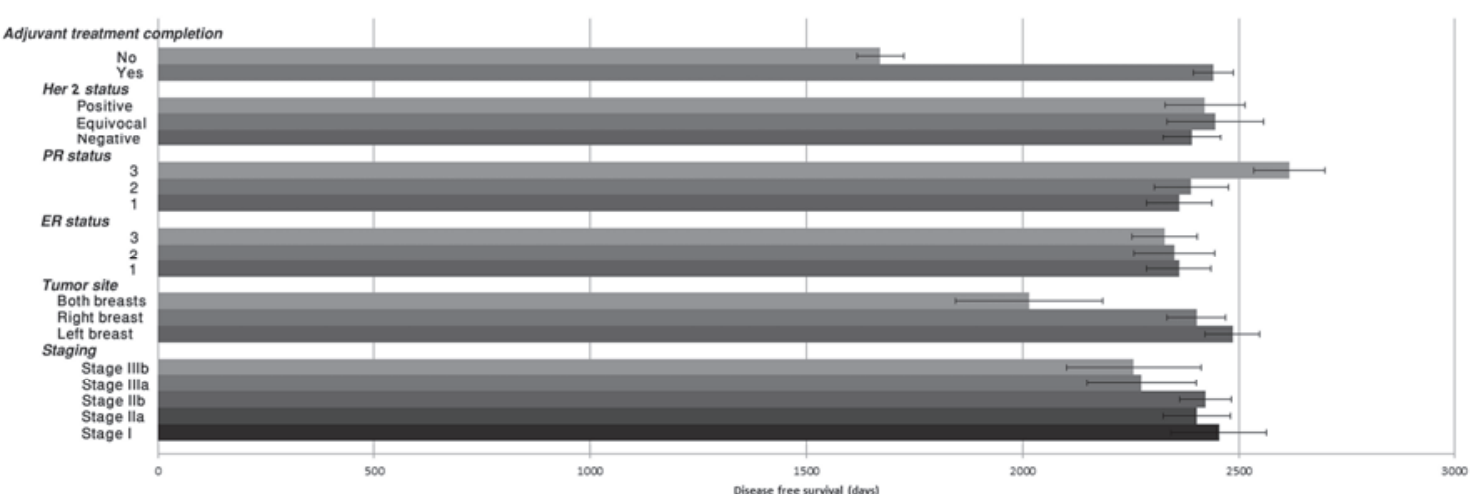

Breast Care 2018;13:121-125 


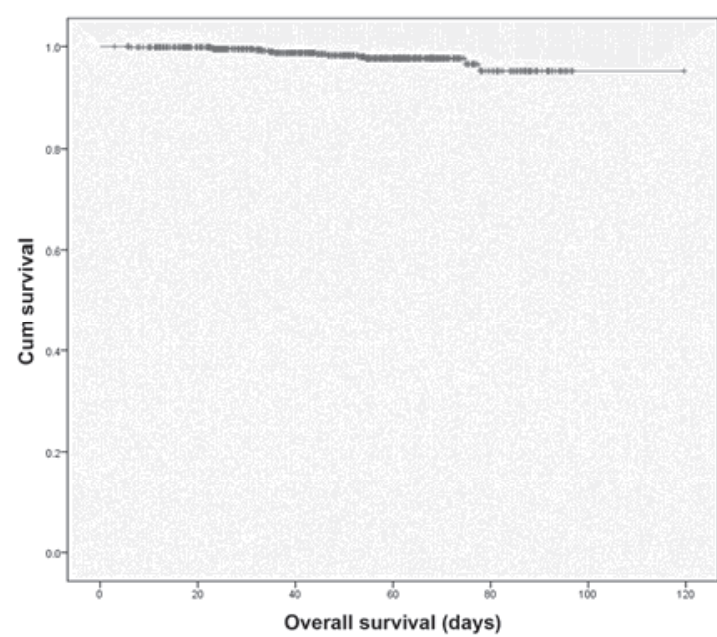

Survival function

Fig. 3. Overall survival assessed by Kaplan-Meier in Lebanese breast cancer patients.

64.0-82.6 months). Mean OS was found to be independent of nodal status; in particular, those with $\mathrm{N} 1$ disease had similar OS compared to those with either N2 or N3 disease $(\mathrm{p}=0.674)$.

\section{Safety Analysis}

Non-hematological adverse events included skin reactions in $32.7 \%$ of the subjects. Multiple adverse events were reported and most commonly included asthenia (66.5\%), alopecia (43.4\%), and diarrhea (24.2\%) (table 3). It is noteworthy that no fatal toxicities occurred. Several hematological adverse events were reported during treatment (table 4). Anemia was the most frequently reported (15.9\%) with grade 3-4 anemia occurring in $4.3 \%$ of patients. Among the 81 (11.6\%) patients who developed mild anemia, the adverse event worsened to moderate anemia in 15 (18.5\%) cases and to severe anemia in 1 case. 59 (8.5\%) patients developed neutropenia. Mild, moderate, and severe neutropenia were reported in 34 (57.6\%), 19 (32.2\%), and 6 (10.2\%) patients, respectively. Among the 35 patients who suffered from mild neutropenia, the adverse event worsened to moderate neutropenia in 2 cases. Among the 24 patients who suffered from moderate neutropenia, the adverse event worsened to severe neutropenia in 2 cases. Febrile neutropenia was encountered in 17 (2.4\%) cases and required hospitalization in 10 cases.

The occurrence of any grade 4 adverse event was found to be significantly more likely $(\mathrm{p}<0.001)$ in patients with an ECOG score of 1 or 2 compared to those with an ECOG score of 0 (odds ratio 10.31; 95\% CI 3.76-28.57), the same trend was observed for the occurrence of any grade 3 adverse event, but this did not reach statistical significance $(\mathrm{p}=0.065)$.

\section{Discussion}

The trials of docetaxel in the adjuvant setting of breast cancer treatment were developed following the demonstration of a survival benefit in the metastatic setting, and have subsequently shown a
Table 3. Overview of non-hematological adverse events of breast cancer patients receiving docetaxel-based treatment

\begin{tabular}{lcl}
\hline Adverse effect & All grades, $\mathrm{n}(\%)$ & Grade 3-4, $\mathrm{n}(\%)$ \\
\hline Skin reactions & $228(32.7)$ & $11(1.6)$ \\
Myalgia & $152(21.8)$ & $72(10.3)$ \\
Asthenia & $464(66.5)$ & $98(14.0)$ \\
Nausea & $119(17.0)$ & $23(3.3)$ \\
Vomiting & $89(12.8)$ & $26(3.7)$ \\
Alopecia & $304(43.4)$ & $52(7.4)$ \\
Sensory neuropathy & $77(11.0)$ & $10(1.4)$ \\
Fluid retention & $76(10.9)$ & $10(1.4)$ \\
Nail changes & $118(16.9)$ & $23(3.3)$ \\
Diarrhea & $169(24.2)$ & $44(6.3)$ \\
\hline
\end{tabular}

Table 4. Overview of hematological adverse events of breast cancer patients receiving docetaxel-based treatment

\begin{tabular}{lll}
\hline Adverse effect & All grades, n (\%) & Grade 3-4, n (\%) \\
\hline Anemia & $111(15.9)$ & $30(4.3)$ \\
Neutropenia & $59(8.5)$ & $24(3.4)$ \\
Leukopenia & $31(4.4)$ & $3(0.4)$ \\
Febrile neutropenia & $17(2.4)$ & $13(1.9)$ \\
Neutrophilia & $1(0.1)$ & $0(0.0)$ \\
Thrombocythemia & $1(0.1)$ & $0(0.0)$ \\
Thrombocytopenia & $1(0.1)$ & $0(0.0)$ \\
\hline
\end{tabular}

significantly longer DFS [3]. We believe that our paper is among the first to definitively report on treatment-related toxicities occurring in a group of patients from a general breast cancer population and compare these toxicities with the available literature.

Real-life experiences with docetaxel-based adjuvant therapy have been rarely reported in the literature. The only available data is limited to a retrospective analysis of 133 patients with early-stage breast cancer [4]. Patients did not benefit from trastuzumab when indicated, and their DFS was limited to 59.7 months. Tumor size and lymph node status were the only independent variables affecting DFS, but our results showed that DFS was independent of all the studied variables and that completion of the planned chemotherapy cycles is the only determining factor of OS [4].

Concerning the toxicity profile of docetaxel, our experience is characterized by a predominance of hematological adverse events (15.9\%), mainly anemia (11.6\%) and neutropenia (8.5\%). Unfortunately, our database does not report on the use of transfusions. Erythrocyte-stimulating agents are not allowed at our institution for patients receiving chemotherapy in the adjuvant setting. On the other hand, the non-hematological toxicity profile of our patients receiving docetaxel differs from that of previously published trials. Skin reactions were as low as $32.7 \%$ with our premedication protocol. Alopecia occurred in $43.4 \%$ of our population, partly due to the preponderant use and high success rate of the scalp cooling system in the vast majority of our patients [5]. Also noteworthy was the absence of any grade 5 docetaxel-related toxicities, which we attribute to the facilitated access of our patients to the treating physician [6]. 
According to our review of the literature, the only other real-life experience of docetaxel in early-stage breast cancer retrospectively reported on the occurrence of febrile neutropenia in a small sample of 101 patients treated with $\mathrm{FE}_{100} \mathrm{C}-\mathrm{D}$ (fluorouracil $500 \mathrm{mg} / \mathrm{m}^{2}$, epirubicin $100 \mathrm{mg} / \mathrm{m}^{2}$, cyclophosphamide $500 \mathrm{mg} / \mathrm{m}^{2} \rightarrow$ docetaxel $\left.100 \mathrm{mg} / \mathrm{m}^{2} \times 3\right)$. The authors compared their findings to those of the PACS 01 trial and demonstrated a higher incidence of adverse events [7]. Our study collected data prospectively which allowed us to audit the eligibility, toxicities, and outcomes of all docetaxelbased regimens. The results of our study allow better transferability of the treatment effect and adverse events to a general populationbased patient group.

In summary, the results of this real-life experience, characterized by a relatively large sample size and long follow-up, confirm that docetaxel is effective and well tolerated in early-stage breast cancer patients. Unfortunately, we did not take into consideration the dose-intensity and schedule (sequential or combined administration) of our docetaxel-based regimens. The toxicity profile of our patients differs from that of the published trials, a finding which should elicit particular interest in the pharmacodynamics of docetaxel in our population. Moreover, oncologists worldwide should carefully observe patients during treatment with docetaxel in order to avoid potential aggravation of adverse events. Such follow-up would reduce the prevalence and severity of docetaxel-related toxicities.

\section{Disclosure Statement}

The authors declare no conflict of interest.

\section{References}

1 Bissery MC, Nohynek G, Sanderink GJ, Lavelle F: Docetaxel (Taxotere): a review of preclinical and clinical experience. Part I: preclinical experience. Anticancer Drugs 1995;6:339-355, 363-368.

2 Pant S, Chilukuri MP, Ramaswamy B: Docetaxel for the post-surgery treatment of patients with node-positive breast cancer. Ther Clin Risk Manag 2008;4:419-424.

3 Martin M, Pienkowski T, Mackey J, et al.; Breast Cancer International Research Group 001 Investigators: Adjuvant docetaxel for node-positive breast cancer. N Engl J Med 2005;352:2302-2313.
4 Azim HA, Abdal-Kader YSED, Mousa MM, Malek RA, Abdalmassih MK, Ibrahim NY: Taxane-based regimens as adjuvant treatment for breast cancer: a retrospective study in Egyptian cancer patients. Asian Pac J Cancer Prev 2015; 16:65-69.

5 Ibrahim T, Kattan J, Assi T, Chahine G, El Karak F, Nasr F, Ghosn M: Efficacy of a silicon based continuous scalp cooling system with thermostat on chemotherapy induced alopecia. J Palliat Care Med 2015;5:209.

6 Assi T, Kattan J, Rassy EE, Tabchi S, Chebib R, Moussa T, Hanna C, El Karak F, Farhat F, Ghosn M: Efficacy and safety of everolimus in hormone receptor positive breast cancer in a developing country: real-life single institutional experience. J Can Res Ther 2017;published online ahead of print. www.cancerjournal.net/ preprintarticle.asp? $i d=183552 ;$ type $=0$.

7 Fraser J, Stelle N, Al Zaman A, Yule A: Are patients in clinical trials representative of the general population? Dose intensity and toxicities associated with FE100C$\mathrm{D}$ chemotherapy in a non-trial population of node positive breast cancer patients compared with PACS01 trial group. Eur J Cancer 2011;47:215-220. 